Supporting information for:

\title{
Understanding How Coacervates Drive Reversible Small Molecule Reactions to Promote Molecular Complexity
}

\author{
Michael I. Jacobs, ${ }^{1}$ Edward R. Jira, ${ }^{1,2}$ and Charles M. Schroeder ${ }^{1,2,3}$ \\ ${ }^{1}$ Beckman Institute for Advanced Science and Technology, University of Illinois at Urbana- \\ Champaign, Urbana, IL 61801 \\ ${ }^{2}$ Department of Chemical and Biomolecular Engineering, University of Illinois at Urbana- \\ Champaign, Urbana, IL 61801 \\ ${ }^{3}$ Department of Materials Science and Engineering, University of Illinois at Urbana-Champaign, \\ Urbana, IL 61801
}

\author{
Contents: \\ Page S-2 Synthesis of amine 1. \\ Synthesis of dye 4. \\ Page S-3 Definition of variables \\ Integrated bimolecular rate law. \\ Page S-4 Reactant quantification equilibrium in PAA-PEG coacervates. \\ Imine synthesis coacervate droplet reaction-diffusion model. \\ Page S-8 Figure S1. Changing imine 3 quantum yield with changing solution composition. \\ Figure S2. Dye 4 molar absorptivity. \\ Page S-9 Figure S3. PAA-PEG coacervate turbidity assay. \\ Figure S4. UV-vis absorption spectra of amine 1, aldehyde $\mathbf{2}$, and dye $\mathbf{4}$. \\ Page S-10 Figure S5. Kinetic traces of imine $\mathbf{3}$ with different starting reactant concentration. \\ Figure S6. $K_{r x n, a q}$ at different concentrations of $\mathrm{NaCl}$. \\ Page S-11 Figure S7. $K_{r x n, a q}$ at different concentrations of PEG and PAA. \\ Figure S8. UV-vis absorption spectra of bulk PAA-PEG coacervate. \\ Page S-12 Figure S9. $K_{r x n, c o a c}$ at different concentrations of NaCl. \\ Figure S10. Partial droplet FRAP measurements. \\ Page S-13 Figure S11. Shift in apparent $K_{r x n, a q}$ versus $f_{\text {coac }}$ at different $r_{\text {coac }}$. \\ Figure S12. ${ }^{1} \mathrm{H}$ NMR spectrum of amine 1. \\ Page S-14 Figure S13. ${ }^{1} \mathrm{H}$ NMR spectrum of molecule 4a \\ Figure S14. ${ }^{1} \mathrm{H}$ NMR spectrum of dye 4. \\ Page S-15 Table S-1. Calculated $\log \left(\underline{K}_{o w}\right)$ of amine 1, aldehyde $\mathbf{2}$, imine $\mathbf{3}$, and dye 4 . \\ Table S-2. Kinetic and transport parameters used in reaction-diffusion model.
}




\section{Experimental methods:}

Materials: Poly(ethylene glycol) $\left(\mathrm{PEG}, \mathrm{M}_{\mathrm{w}} \approx 35 \mathrm{kDa}\right.$ ) was purchased from Sigma Aldrich. Poly(acrylic acid) (PAA, $\mathrm{M}_{\mathrm{w}} \approx 240 \mathrm{kDa}$ ) was purchased from Alfa-Aesar. N-Methyl-N-(2hydroxyethyl)-4-aminobenzaldehyde 1 was purchased from AA Blocks LLC and used without further purification. N-Methyl-2-amino-benzothiazolium salt 1 was synthesized as previously described. ${ }^{1,2}$ The kinetically stable dye analog (4) of imine 3 (which replaces the imine functional group with an alkene) was synthesized as previously described. ${ }^{3}$ All solutions were prepared in 50 $\mathrm{mM}$ citrate buffer, $\mathrm{pH} 3$.

Synthesis of amine 1: 2-Aminobenzthiazole (3.5 g, $23 \mathrm{mmol})$ was dissolved in acetonitrile (50 $\mathrm{mL}$ ), and iodomethane $(5 \mathrm{~g}, 35 \mathrm{mmol})$ was added. The solution was stirred at $45{ }^{\circ} \mathrm{C}$ for 16 hours. After cooling to room temperature, $50 \mathrm{~mL}$ of ethyl ether were added, and the white suspension was filtered. The precipitate was washed with diethyl ether and dried under vacuum. Amine 1 was obtained as an off-white pure solid (4.1 g, $14 \mathrm{mmol}, 61 \%) .{ }^{1} \mathbf{H} \mathbf{~ N M R}\left(500 \mathrm{MHz},\left(\mathrm{CD}_{3}\right)_{2} \mathrm{CO}\right) \delta 9.89$ $(\mathrm{s}, 2 \mathrm{H}), 8.04(\mathrm{~d}, J=7.9 \mathrm{~Hz}, 1 \mathrm{H}), 7.76(\mathrm{~d}, J=8.4 \mathrm{~Hz}, 1 \mathrm{H}), 7.65$ (t, $J=7.9 \mathrm{~Hz}, 1 \mathrm{H}), 7.50(\mathrm{t}, J=7.9$ $\mathrm{Hz}, 1 \mathrm{H}), 4.03$ (s, 3H) (Figure S9)

Synthesis of dye 4: Dye 4 was synthesized in two steps from commercially available 2methylbenzothiazole and aldehyde 2 according to the steps below.

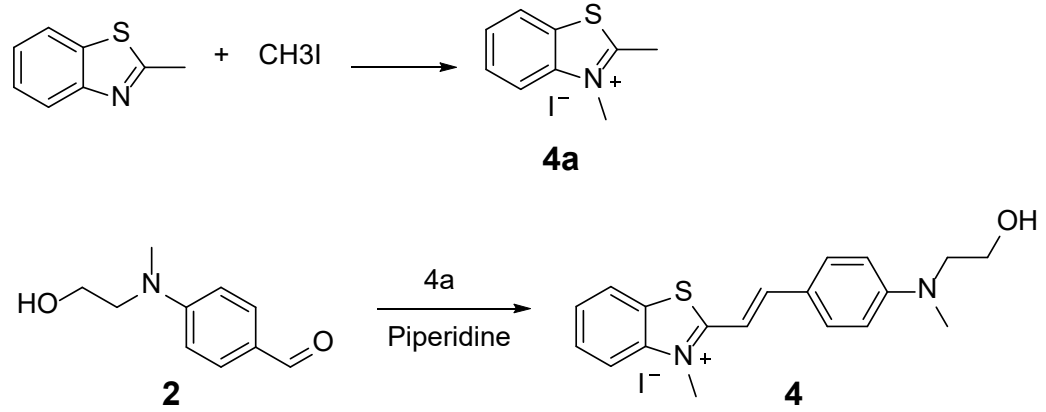

2-Methylbenzothiazole (3.5 g, $23 \mathrm{mmol})$ was dissolved in $50 \mathrm{~mL}$ acetonitrile, and iodomethane (5 $\mathrm{g}, 35 \mathrm{mmol}$ ) was added. The solution was heated to $45^{\circ} \mathrm{C}$ for 18 hours. After cooling to room temperature, $75 \mathrm{~mL}$ of ethyl ether were added, and the suspension was filtered. The precipitate was washed with diethyl ether and dried under vacuum. 2,3-Dimethylbenzothiazolium iodide (4a) was obtained as an off-white powder $(1.1 \mathrm{~g}, 4 \mathrm{mmol}, 17 \%)$. ${ }^{1} \mathbf{H}$ NMR $\left(500 \mathrm{MHz},\left(\mathrm{CD}_{3}\right)_{2} \mathrm{SO}\right) \delta 8.43$ $(\mathrm{d}, J=8.2 \mathrm{~Hz}, 1 \mathrm{H}), 8.29(\mathrm{~d}, J=8.5 \mathrm{~Hz}, 1 \mathrm{H}), 7.90(\mathrm{t}, 7.8 \mathrm{~Hz}, 1 \mathrm{H}), 7.81(\mathrm{t}, J=7.7 \mathrm{~Hz}, 1 \mathrm{H}), 4.20(\mathrm{~s}$, $3 \mathrm{H}), 3.16(\mathrm{~s}, 3 \mathrm{H})$ (Figure S10).

Using a $250-\mathrm{mL}$ round bottom flask with a condenser, $0.58 \mathrm{~g}(2.0 \mathrm{mmol})$ of compound $4 \mathrm{a}$, $0.37 \mathrm{~g}(2.1 \mathrm{mmol})$ of aldehyde 2 , and $60 \mathrm{~mL}$ of ethanol were mixed. Ten drops of piperidine were added to the mixture, and the solution was heated to reflux for $4 \mathrm{hr}$. After cooling to room temperature, $60 \mathrm{~mL}$ of ethyl ether were added over the course of $10 \mathrm{~min}$. The solution was filtered, and the precipitate was washed with ethyl ether and dried under vacuum. Dye 4 was obtained as a deep purple solid $(0.47 \mathrm{~g}, 1.0 \mathrm{mmol}, 50 \%) .{ }^{1} \mathbf{H}$ NMR: $\left(500 \mathrm{MHz},\left(\left(\mathrm{CD}_{3}\right)_{2} \mathrm{SO}\right) \delta 8.29(\mathrm{~d}, J=8.1\right.$ $\mathrm{Hz}, 1 \mathrm{H}), 3.65-3.52(\mathrm{~m}, 2 \mathrm{H}), 7.89(\mathrm{~d}, J=8.7 \mathrm{~Hz}, 2 \mathrm{H}) 7.78(\mathrm{t}, J=7.9 \mathrm{~Hz}, 1 \mathrm{H}), 7.68(\mathrm{t}, J=7.8 \mathrm{~Hz}$, $1 \mathrm{H}), 7.61(\mathrm{~d}, J=15.3 \mathrm{~Hz}, 1 \mathrm{H}), 6.87(\mathrm{~d}, J=6.9 \mathrm{~Hz}, 2 \mathrm{H}), 4.81(\mathrm{t}, J=5.2 \mathrm{~Hz}, 1 \mathrm{H}), 4.22(\mathrm{~s}, J=\mathrm{Hz}$, $3 \mathrm{H}), 3.60(\mathrm{~m}, 4 \mathrm{H}), 3.12(\mathrm{~s}, 3 \mathrm{H})$ (Figure S11). 


\section{Definition of variables:}

\begin{tabular}{c|l} 
Parameter & Definition \\
\hline$K_{r x n}$ & Reaction equilibrium constant \\
$K_{r x n, a q}$ & Reaction equilibrium constant in aqueous phase \\
$K_{r x n, c o a c}$ & Reaction equilibrium constant in coacervate phase \\
$k_{f}$ & Forward reaction rate constant \\
$k_{r}$ & Reverse reaction rate constant \\
$K_{\text {part }}$ & Partitioning coefficient \\
$V_{t o t}$ & Total volume of coacervate droplets and aqueous solution \\
$V_{c o a c}$ & Volume of coacervate \\
$V_{a q}$ & Volume of aqueous solution \\
$f_{\text {coac }}$ & Volume fraction of coacervate \\
$D_{\text {im }}$ & Imine $\mathbf{3}$ diffusion coefficient in coacervates \\
{$[\text { imine }]_{\mathrm{eq}, \mathrm{part}}$} & Coacervate imine $\mathbf{3}$ concentration at partitioning equilibrium (from experiment) \\
{$[\text { imine }]_{\mathrm{eq}, \text { rxn }}$} & Coacervate imine $\mathbf{3}$ concentration at reaction equilibrium (from experiment) \\
{$[\text { imine }]_{\mathrm{eq}, \text { sim }}$} & Coacervate imine $\mathbf{3}$ concentration at equilibrium (from simulations) \\
{$[\text { imimine }]_{\mathrm{aq}, \mathrm{eq}}$} & Aqueous imine $\mathbf{3}$ equilibrium at reaction equilibrium (from experiment) \\
{$[\text { imine }]_{\mathrm{aq}, \text { sim }}$} & Aqueous imine $\mathbf{3}$ concentration at equilibrium (from simulations)
\end{tabular}

Integrated bimolecular rate law: An integrated bimolecular rate law was used to determine the forward $k_{f}$ and reverse $k_{r}$ rate constants from the fluorescence data. Imine synthesis between amine $\mathbf{1}$ and aldehyde $\mathbf{2}$ is a bimolecular reversible reaction. A generic reversible bimolecular reaction between reactants amine $\mathbf{1}(\mathrm{Am})$ and aldehyde $\mathbf{2}$ (Ald) to produce imine $\mathbf{3}(\mathrm{Im})$ can be written as:

$$
A m+A l d \rightleftarrows I m ; K_{r x n}=\frac{k_{f}}{k_{r}} ; \frac{d[I m]}{d t}=k_{f}\left([A m][A l d]-\frac{[I m]}{K_{r x n}}\right),
$$

where $K_{r x n}$ is the reaction equilibrium constant, $k_{f}$ is the forward rate constant, and $k_{r}$ is the reverse rate constant. An integrated rate law for the reversible formation of imine 3 is written as: ${ }^{4}$

$$
\begin{aligned}
& {[\mathrm{Im}]_{t}=[\mathrm{Im}]_{e}+\frac{\left([\mathrm{Im}]_{0}-[\mathrm{Im}]_{e}\right) \alpha E}{1+\left([\mathrm{Am}]_{e}-[\mathrm{Am}]_{0}\right) E}} \\
& \alpha=[\mathrm{Am}]_{e}+[\mathrm{Ald}]_{e}+1 / K_{r x n} \approx 1 / K_{r x n} \\
& \beta=\ln \left([\mathrm{Am}]_{0}+[\mathrm{Ald}]_{e}+1 / K_{r x n}\right) \approx \ln \left(1 / K_{r x n}\right) \\
& E=e^{-\left(k_{f} \alpha t+\beta\right)} \approx K_{r x n} e^{-\frac{k_{f}}{K_{r x n}} t}
\end{aligned}
$$

For the reaction conditions in our experiments $\left([\mathrm{Im}]_{0}=0,[\mathrm{Am}]_{\mathrm{e}} \approx[\mathrm{Am}]_{0},[\mathrm{Ald}]_{\mathrm{e}} \approx[\mathrm{Ald}]_{0}, K_{r x n}<<\right.$ 1 ), expressions for $\alpha, \beta$, and $E$ can be simplified as shown in the equations above. Incorporating these simplifications into Eq. S2 yields:

$$
[\operatorname{Im}]_{t}=[\operatorname{Im}]_{e}\left(1-e^{-\frac{k_{f}}{K_{r x n}} t}\right) .
$$

Using the expressions shown in Eq. S1, this expression can be rewritten to incorporate the initial reactant concentrations: 


$$
[\mathrm{Im}]_{t}=K_{r x n}[A m]_{0}[\mathrm{Ald}]_{0}\left(1-e^{-k_{r} t}\right),
$$

which is the expression used to describe the concentration of imine $\mathbf{3}$ as a function of time in the solution and coacervate phases. For reversible bimolecular reactions, $k_{r}$ and $k_{f}$ are related by:

$$
k_{r}=\frac{k_{f}}{K_{r x n}} .
$$

Thus, $k_{f}$ was calculated from $K_{r x n}$ and $k_{r}$, and the reaction was well characterized in bulk solution.

Reactant quantification equilibrium in PAA-PEG coacervates: Reactant concentrations in the coacervate were determined from the total reactant concentrations in the cuvette [Reactant $]_{\text {total }}$, their experimentally measured partitioning coefficients $K_{\text {part }}$, the total volume of solution $V_{\text {tot }}$, and measured volume of the coacervate $V_{\text {coac }}$ according to:

$$
[\text { Reactant }]_{\text {coac }}=[\text { Reactant }]_{\text {tot }} V_{\text {tot }} /\left(V_{\text {coac }}+\frac{V_{\text {tot }}-V_{\text {coac }}}{K_{\text {part }}}\right) .
$$

Because $K_{r x n, c o a c}<<1$, the concentrations of amine $\mathbf{1}$ and aldehyde $\mathbf{2}$ do not significantly change as the reaction approaches equilibrium.

Imine synthesis coacervate droplet reaction-diffusion model: We developed a reactiondiffusion model to describe reaction kinetics within a coacervate droplet suspended in a wellmixed aqueous phase. Given the spherical shape of the coacervate droplet, we assume a spherical coordinate system for this problem. To proceed, we make several assumptions about the system:

1. Spherical symmetry. With the origin of the coordinate system located at the center of a suspended coacervate droplet, we assume that the concentration of all species is spherically symmetric such that [molecule] $(\mathrm{r}, \theta, \varphi, \mathrm{t})=$ [molecule] $(\mathrm{r}, \mathrm{t})$, where [molecule] is the concentration of either amine $\mathbf{1}$, aldehyde $\mathbf{2}$, or imine $\mathbf{3}$. Therefore, the only independent variables are radial position $r$ and time $t$.

2. Constant density. It is assumed that the densities of the coacervate phase and aqueous phase are constant.

3. Constant diffusion coefficients. It is assumed that the diffusivity of molecular species $D_{i}$ is constant with respect to time and spatial position within the coacervate droplet.

4. Quiescent system (no flow). It is assumed that there is no bulk convection or flow in the system.

5. Chemical reaction. It is assumed that the imine synthesis reaction proceeds according to Eq. S1 in both the coacervate droplet and the surrounding aqueous phase according to measured kinetic parameters as described in the main text and summarized below in Table S2.

6. Constant reaction concentration. Because $K_{r x n}<<1$ in both aqueous solution and the coacervate droplet, the concentration of aldehyde $\mathbf{1}$ and amine $\mathbf{2}$ are assumed to be constant (fixed by their partition coefficients) in the reaction diffusion model.

7. Well-mixed aqueous phase. Because the diffusion coefficients within the aqueous phase are several orders of magnitude larger than that in the coacervate droplet, ${ }^{8}$ changes in 
aqueous concentration due to diffusional flux into or out of the coacervate droplet are distributed evenly throughout the aqueous phase.

First, we describe the reaction-diffusion within the coacervate droplet. The only diffusing chemical species with changing concentration within the coacervate droplet is imine 3 . The transport and reaction of imine 3 within the coacervate droplet of radius $R_{\text {coac }}$ can be modeled by the reactiondiffusion equation in spherical coordinates according to:

$$
\frac{\partial[\mathrm{Im}]_{\text {coac }}}{\partial t}=D_{I m}\left(\frac{1}{r^{2}} \frac{\partial}{\partial r}\left(r^{2} \frac{\partial[\mathrm{Im}]_{\text {coac }}}{\partial r}\right)\right)+k_{f, \text { coac }}\left([\mathrm{Am}]_{\text {coac }}[A l d]_{\text {coac }}-\frac{[\mathrm{Im}]}{K_{r x n, \text { coac }}}\right),
$$

where $[\mathrm{Im}]_{\mathrm{coac}},[\mathrm{Am}]_{\mathrm{coac}}$, and [Ald $]_{\mathrm{coac}}$ are the molar concentrations of imine $\mathbf{3}$, amine $\mathbf{1}$ (constant), and aldehyde 2 (constant), respectively. $D_{I m}$ is the diffusion coefficient of imine $\mathbf{3}$ within the coacervate phase. This equation is considered in the coacervate phase for $0 \leq r \leq R_{\text {coacc }}$. This secondorder partial differential equation requires one initial condition and two boundary conditions. The initial condition:

$$
[\operatorname{Im}](r, t=0)=0,
$$

implies that there is no imine $\mathbf{3}$ at the start of the simulation. The boundary condition at the coacervate-aqueous interface is given by:

$$
[\operatorname{Im}]_{\text {coac }}\left(r=R_{\text {coac }}, t\right)=K_{\text {part }, \text { im }}[\operatorname{Im}]_{a q}(t),
$$

where $K_{\text {part, im }}$ is the partitioning coefficient of imine 3 within the PAA-PEG coacervate. The value of $K_{\text {part }, i m}$ changes with concentration of $\mathrm{NaCl}$ (Table S2). For this boundary condition, we assume equality of flux of imine $\mathbf{3}$ across the boundary:

$$
\left.J_{i m}\right|_{r=R} ^{c o a c}=\left.J_{i m}\right|_{r=R} ^{a q}
$$

where $J$ is the molar flux of imine 3 . The second boundary condition is given by symmetry at the origin:

$$
\left.\frac{\partial[\mathrm{Im}]_{\text {coac }}(t)}{\partial r}\right|_{r=0}=0 .
$$

The concentration of imine $\mathbf{3}$ evolves in the aqueous phase according to:

$$
\frac{\partial[I m]_{a q}}{\partial t}=k_{f, a q}\left([A m]_{a q}[A l d]_{a q}-\frac{[I m]_{a q}}{K_{r x n, a q}}\right)+\frac{J_{i m}}{V_{a q}}
$$

The first expression describes the imine synthesis reaction kinetics within aqueous solution using kinetic parameters listed in Table S2. The second expression distributes the molar flux of imine $\mathbf{3}$ from the boundary condition in Eq. S9 across the entire aqueous volume. The aqueous volume is calculated according to the inputted volume fraction $f_{\text {coac }}$ according to: 


$$
V_{a q}=V_{\text {coac }} \frac{1-f_{\text {coac }}}{f_{\text {coac }}},
$$

where $V_{\text {coac }}$ is the coacervate volume $\left(4 / 3 \pi R_{\text {coac }}{ }^{3}\right)$. The aqueous concentration of amine and aldehyde $\left([\text { Reactant }]_{\mathrm{aq}}\right)$ are determined from the inputted total reactant concentration [reactant $]_{\text {total }}$ according to:

$$
[\text { Reactant }]_{a q}=[\text { Reactant }]_{\text {total }} \frac{V_{\text {coac }}+V_{a q}}{V_{\text {coac }} K_{\text {part,reactant }}+V_{a q}},
$$

where $K_{\text {part, reactant }}$ is the partitioning coefficient of either amine $\mathbf{1}\left(K_{\text {part,am }}\right)$ or aldehyde $\mathbf{2}\left(K_{\text {part,ald }}\right)$ given in Table S2. The concentration of reactants in the coacervate phase is given by the product of the aqueous reactant concentration and the reactant partitioning coefficient (Table S2). As described in the initial assumptions, this concentration is constant with time due to the small $K_{r x n}$ within both the aqueous and coacervate phases.

Eqs. S7 and S12 were numerically solved to describe the radial concentration profile of imine 3 within the coacervate solution over time. We used Python for numerical calculations. Utilizing the finite difference method, the region within the coacervate droplet from 0 to $R_{\text {coac }}$ was divided into $n+1$ layers with equal thickness $\Delta r$, where $r_{n+1}$ represents the boundary condition and $r_{i}=i \Delta r(i=1,2,3, \ldots, \mathrm{n})$ represents any position between the center and the boundary of the droplet. The concentration imine 3 as a function of position and time $t$ is written as [im] $]_{i, t}$. Layer thicknesses of $0.2 \mu \mathrm{m}$ and time steps $(\Delta t)$ of $100 \mu$ s were reasonable to ensure accuracy of numerical calculations. The concentration of imine $\mathbf{3}$ in a given layer at a given time is given by:

$$
[\mathrm{im}]_{i, t+\Delta t}=[\mathrm{im}]_{i, t}+\frac{\partial[\mathrm{im}]_{i, t}}{\partial t} \Delta t .
$$

Eq. S7 can be expressed as:

$$
\begin{array}{r}
\frac{\partial[i m]_{i, t}}{\partial t}=D_{i m}\left(\frac{[i m]_{i+1}-2[i m]_{i}+[i m]_{i-1}}{(\Delta r)^{2}}+\frac{2}{i \Delta r} \frac{[i m]_{i+1}-[i m]_{i}}{\Delta r}\right) \\
+k_{f, \text { coac }}\left([\mathrm{Am}]_{\text {coac }}[\text { Ald }]_{\text {coac }}-\frac{[i m]_{i, t}}{K_{\text {rxn }, \text { coac }}}\right)
\end{array}
$$

To calculate the aqueous concentration of imine 3 over time ([Im $]_{\text {aq, }, t)}$, Eq. S12 is used in Eq. S15 after calculating the necessary molar flux of imine $\mathbf{3}(\mathrm{J} \mathrm{im})$ between the outermost coacervate layer and aqueous solution to maintain the boundary condition in Eq. S9. Thus, from the initial boundary condition, the radial concentration of imine $\mathbf{3}$ within the coacervate and in aqueous solution could be modeled over time. Typical simulations to measure radial imine $\mathbf{3}$ concentrations at equilibrium were run for $900 \mathrm{~s}$.

\section{References:}

1 K. Meguellati, M. Spichty and S. Ladame, Org. Lett., 2009, 11, 1123-1126.

2 K. Meguellati, A. Fallah-araghi, J. Baret and E. Harrak, 2013, 11332-11334.

3 D. Li, X. Tian, A. Wang, L. Guan, J. Zheng, F. Li, S. Li, H. Zhou, J. Wu and Y. Tian, Chem. Sci., 2016, 7, 2257-2263.

$4 \quad$ J. R. Pladziewicz, J. S. Lesniak and A. J. Abrahamson, J. Chem. Educ., 1986, 63, 850851. 
5 T. Z. Jia, C. Hentrich and J. W. Szostak, Orig. Life Evol. Biosph., 2014, 44, 1-12.

6 D. Axelrod, D. E. Koppel, J. Schlessinger, E. Elson and W. W. Webb, Biophys. J., 1976, 16, 1055-1069.

7 B. L. Sprague, R. L. Pego, D. A. Stavreva and J. G. McNally, Biophys. J., 2004, 86, 34733495.

8 K. R. Wilson, A. M. Prophet, G. Rovelli, M. D. Willis, R. J. Rapf and M. I. Jacobs, Chem. Sci., 2020, 11, 8533-8545.

9 I. V. Tetko, J. Gasteiger, R. Todeschini, A. Mauri, D. Livingstone, P. Ertl, V. A. Palyulin, E. V. Radchenko, N. S. Zefirov, A. S. Makarenko, V. Y. Tanchuk and V. V. Prokopenko, J. Comput. Aided. Mol. Des., 2005, 19, 453-463. 


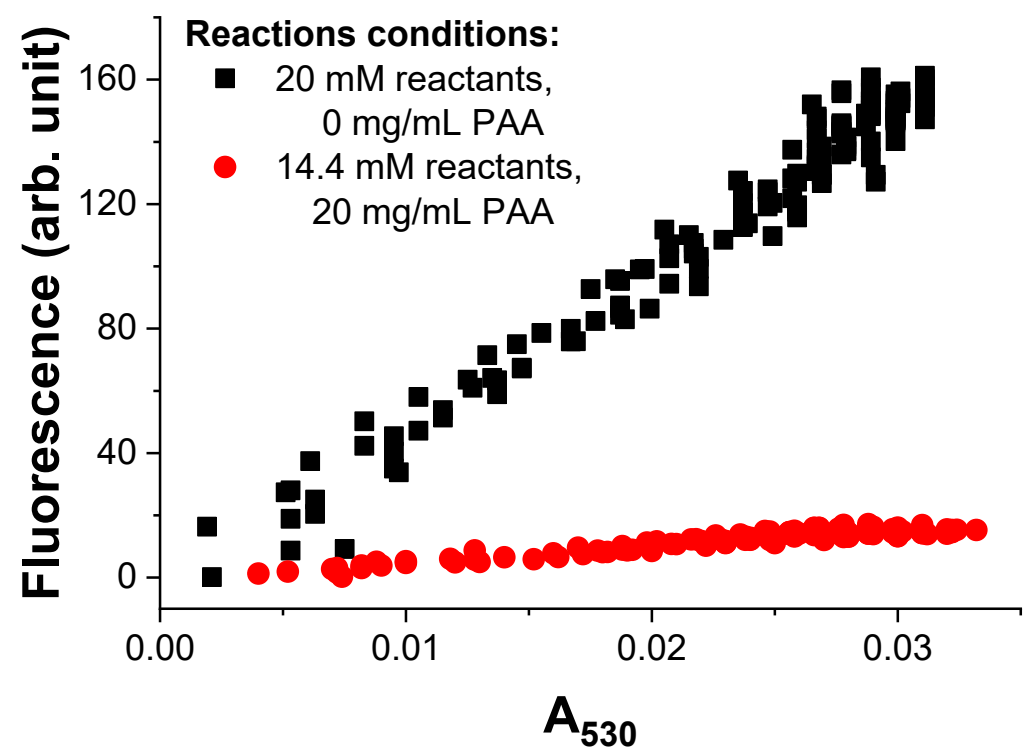

Figure S1. Both absorption and fluorescence were measured over time during synthesis of imine 3 at different solution conditions. The fluorescence intensity of imine $3\left(\lambda_{\mathrm{ex}}=515 \mathrm{~nm}, \lambda_{\mathrm{em}}=550\right.$ $\mathrm{nm}$ ) is plotted against absorbance at $530 \mathrm{~nm}$. Quantum yield is proportional to the slope of fluorescence intensity versus absorption. Comparing the slopes here, the quantum yield of imine 3 in $20 \mathrm{mg} / \mathrm{mL}$ PAA is $10 \mathrm{x}$ larger than that in $0 \mathrm{mg} / \mathrm{mL}$ PAA, demonstrating fluorescence from imine 3 is sensitive to chemical environment.

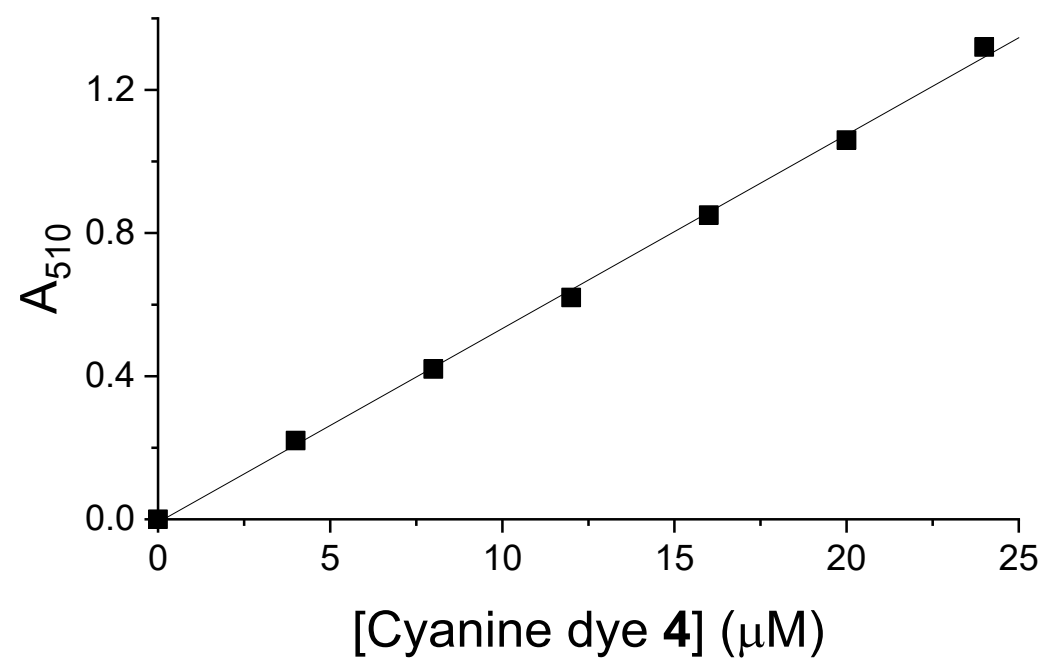

Figure S2. Absorption of dye $\mathbf{4}$ at $510 \mathrm{~nm}$ versus dye concentration. Based on these experiments, the molar absorptivity in water was determined to be $\varepsilon_{510}=(5.42 \pm 0.09) \times 10^{4} \mathrm{~cm}^{-1} \mathrm{~s}^{-1}$. 


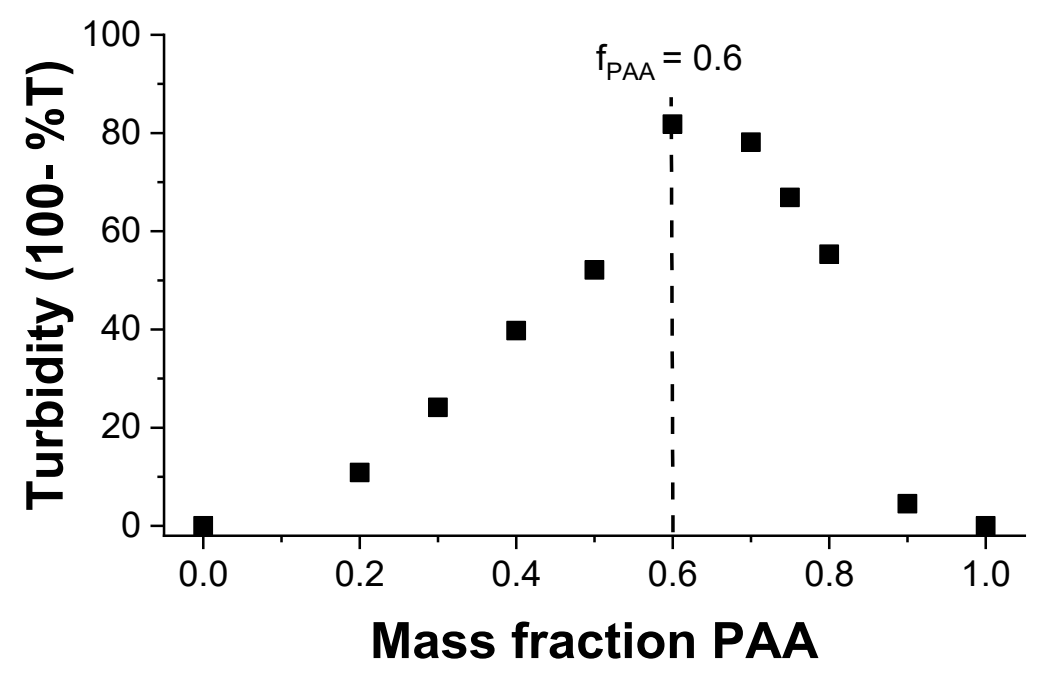

Figure S3. Turbidity of PAA-PEG coacervate ([macromolecule $\left.]_{\text {total }}=0.25 \mathrm{mg} / \mathrm{mL}\right)$ vs. mass fraction of PAA $\left(f_{P A A}\right)$. The highest turbidity was observed at $f_{P A A}=0.6$, which was used throughout experimentation.
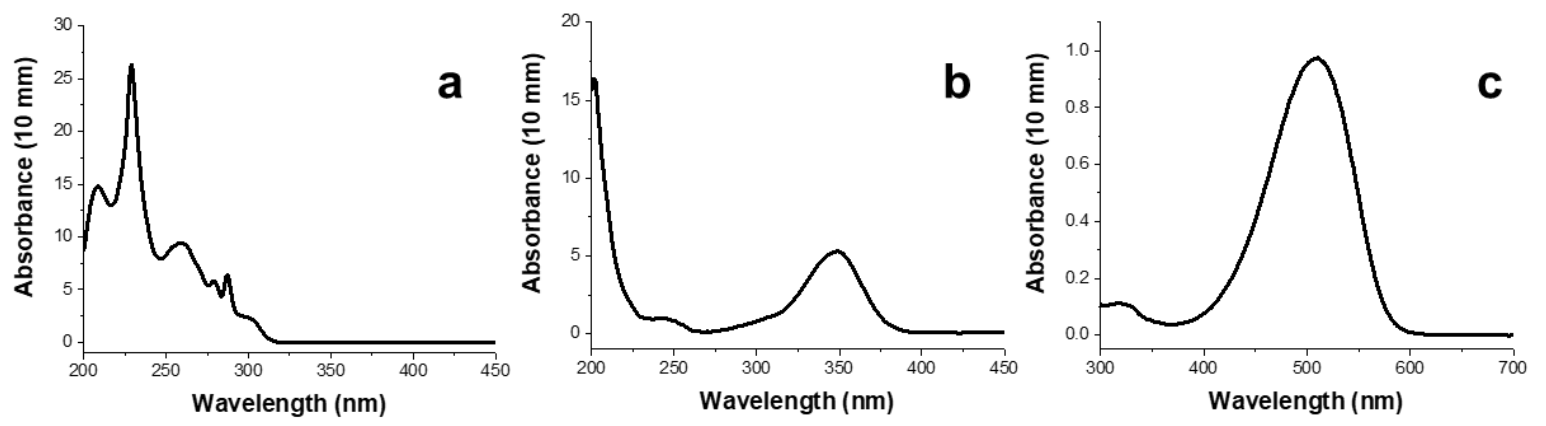

Figure S4. UV-vis absorption spectra of (a) amine 1 (4 mM), (b) aldehyde 2 (0.6 mM), and (c) dye $4(20 \mu \mathrm{M})$. 


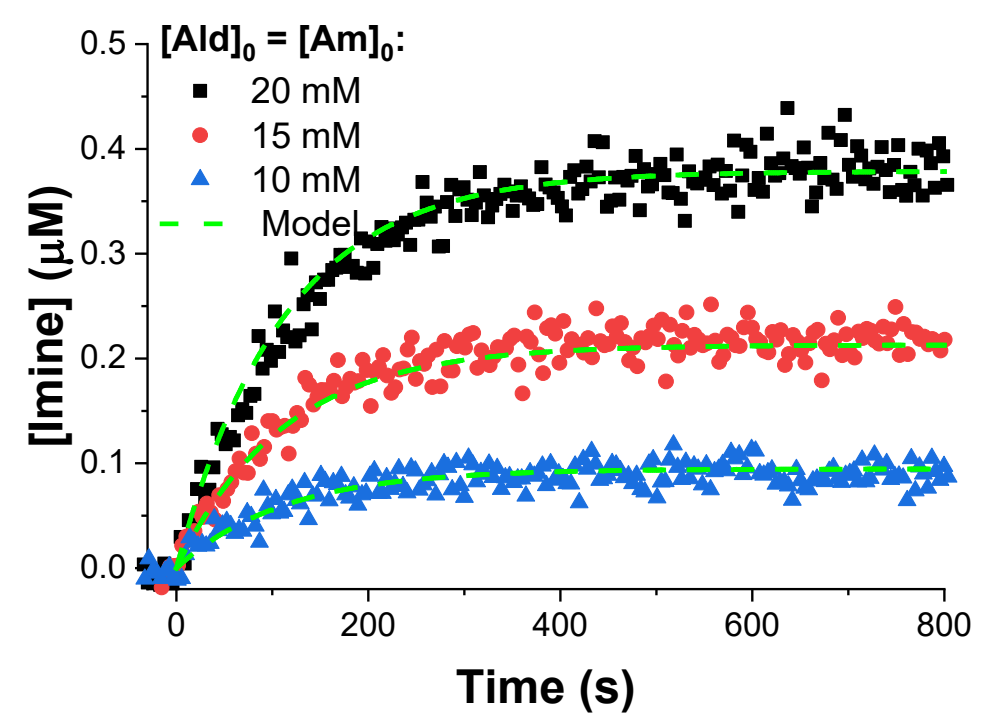

Figure S5. Aqueous imine 3 concentration versus time in $50 \mathrm{mM}$ citrate buffer $(\mathrm{pH} 3)$ and $0 \mathrm{M}$ $\mathrm{NaCl}$. Concentrations are determined from calibrated fluorescence intensities. The dashed green line shows the fit to the reversible bimolecular kinetics model (Eq. S4).

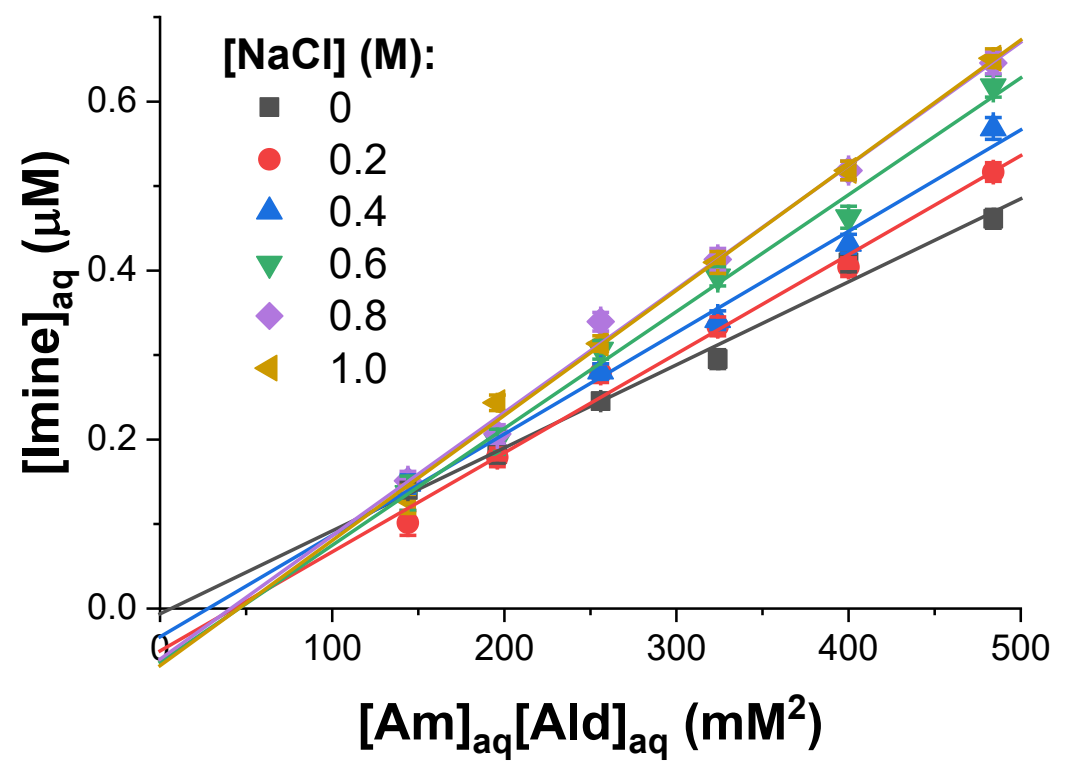

Figure S6. Equilibrium aqueous imine 3 concentration versus the product of reactant concentrations at different solution concentrations of $\mathrm{NaCl}$. The slope was used to calculate $K_{r x n, a q}$. 


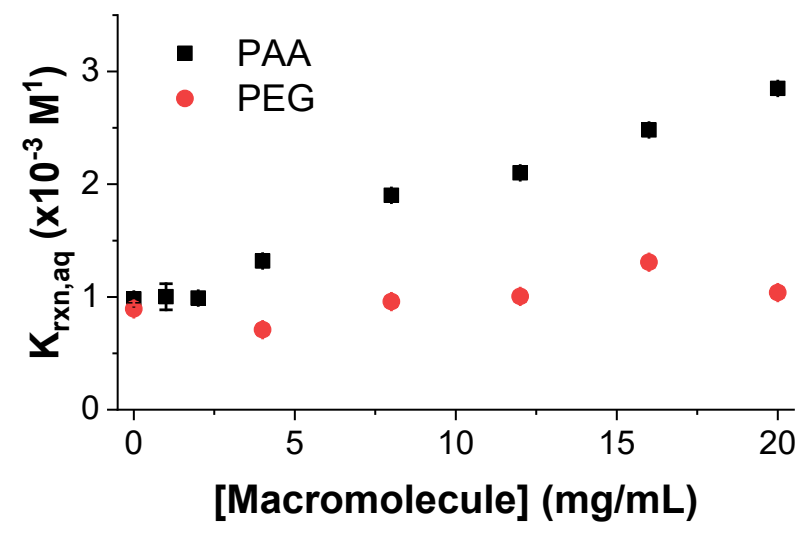

Figure S7. Imine synthesis reaction equilibria versus the concentration of PAA or PEG. Reaction equilibrium is shifted to favor product formation at higher PAA concentrations. In general, PEG does not shift reaction equilibrium.
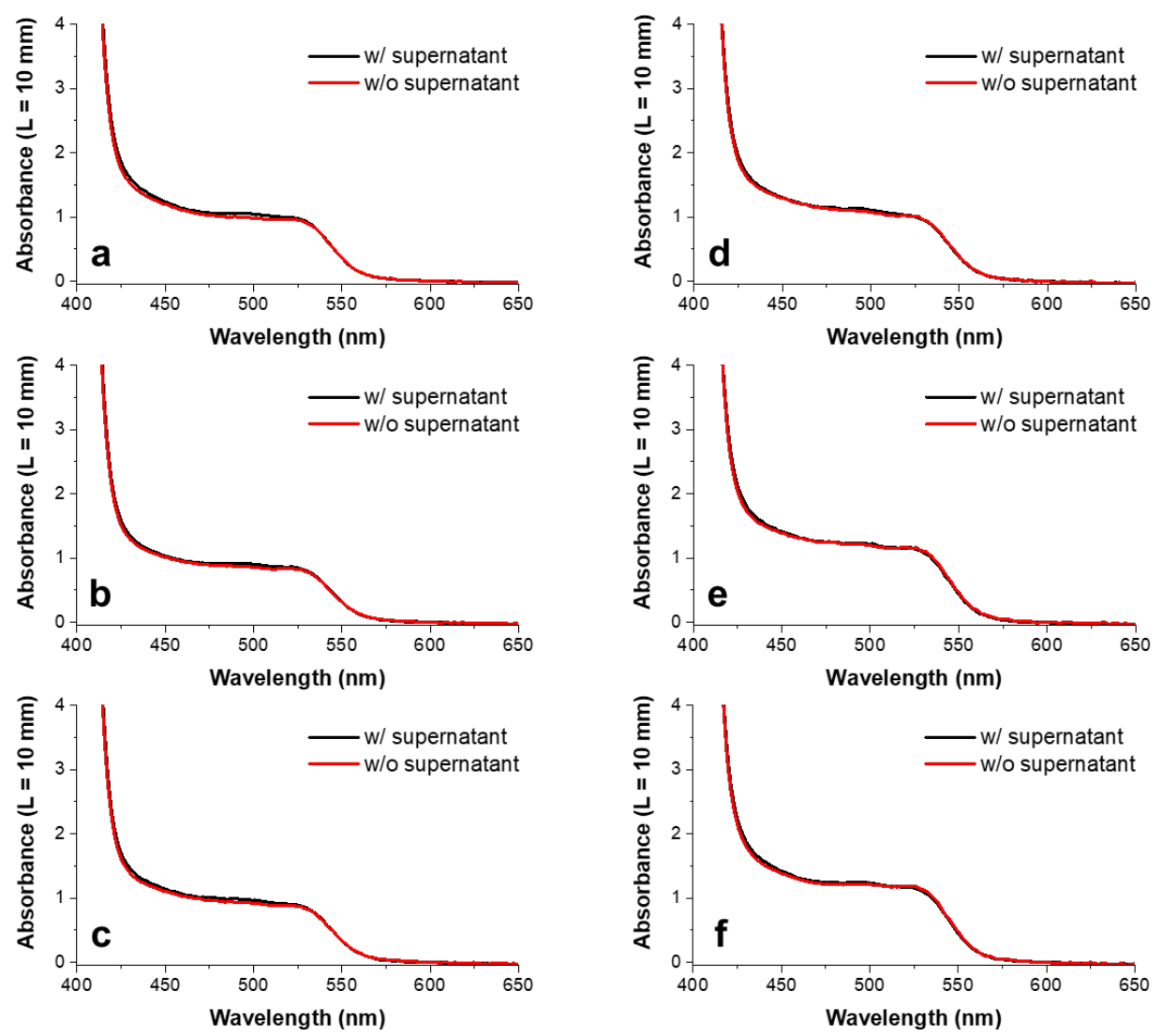

Figure S8. UV-vis spectra of bulk coacervate after equilibrating for several days both with and without the aqueous supernatant present. Samples were collected at (a) 0, (b) 0.2, (c) 0.4, (d) 0.6, (e) 0.8 , and (f) $1.0 \mathrm{M} \mathrm{NaCl}$. Our results show no changes in imine 3 concentration between samples with and without the supernatant because the reacto-diffusive length within the coacervate is much smaller than the characteristic size of the sample. 


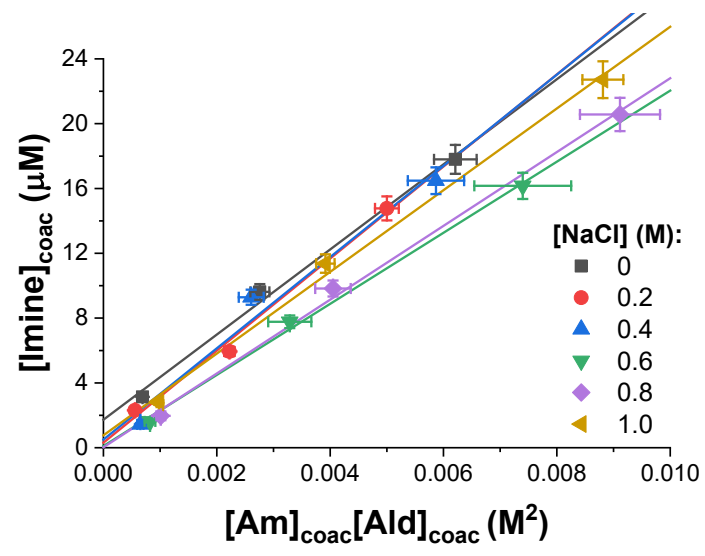

Figure S9. Equilibrium coacervate imine 3 concentration versus the product of reactant concentrations at different concentrations of $\mathrm{NaCl}$. The slope was used to determine $K_{\text {rxn,coac }}$. Overall, our results show no obvious trend with increasing [ $\mathrm{NaCl}]$.
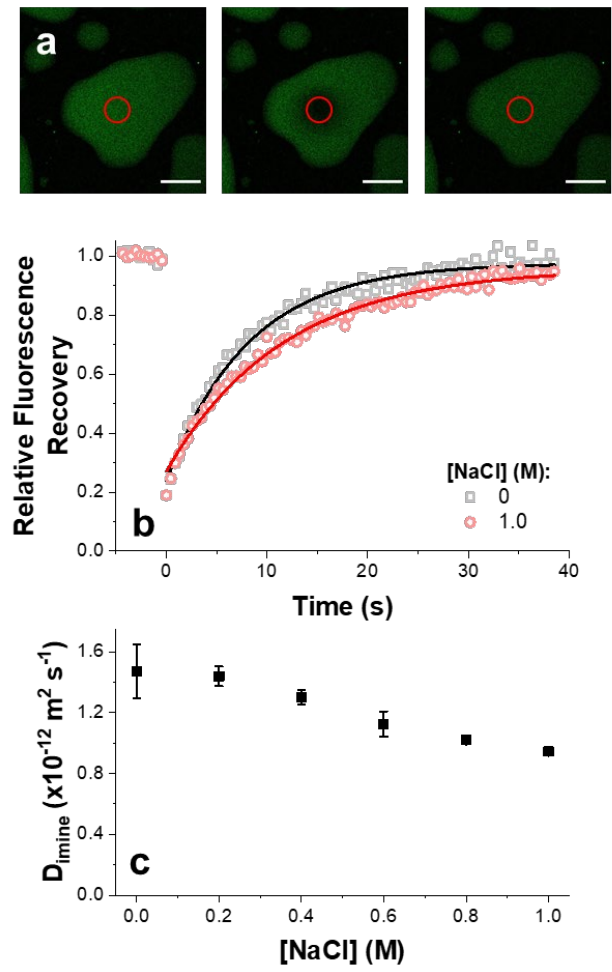

Figure S10. Partial droplet FRAP of imine 3 within PAA-PEG coacervate droplets to determine $D_{i m}$. (a) Images of fluorescence recovery of a non-immersed PAA-PEG coacervate droplet (from $2 \mathrm{mg} / \mathrm{mL}$ macromolecule, $f_{P A A}=2 \mathrm{mg} / \mathrm{mL}$ ) at $0 \mathrm{M} \mathrm{NaCl}$. The scale bar represents $20 \mu \mathrm{m}$. (b) Fluorescence recovery traces of a droplet from $0 \mathrm{M}$ and $1.0 \mathrm{M} \mathrm{NaCl}$. (c) Imine diffusion constant in PAA-PEG coacervates at different $\mathrm{NaCl}$ concentrations. 


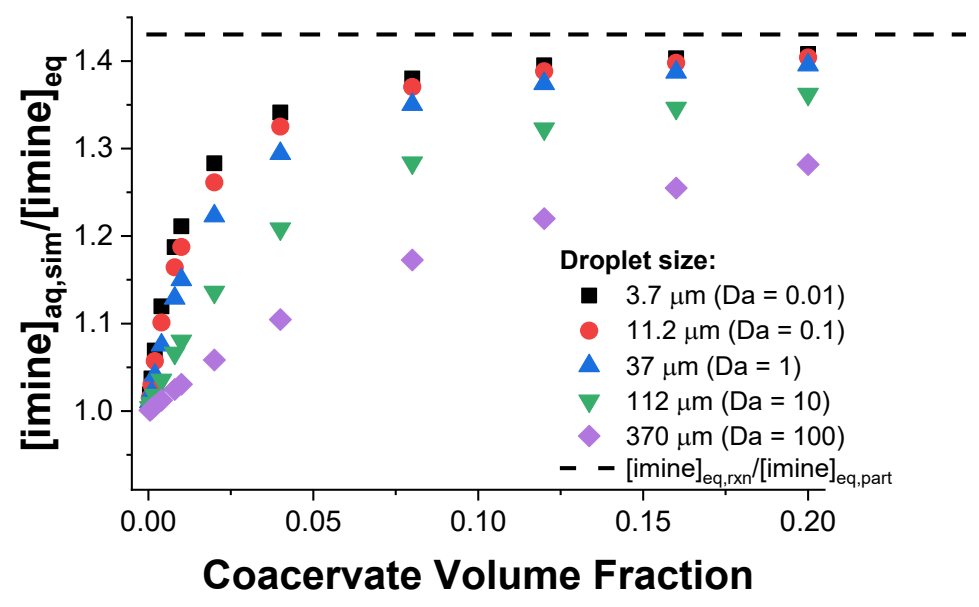

Figure S11. Simulated shifts in aqueous reaction equilibrium at different coacervate volume fractions. The dashed line represents the asymptotic shift in aqueous reaction equilibrium where coacervate reaction and partition equilibrium predict the same concentration of imine 3 . At larger droplet sizes, it takes larger volume fractions to reach this asymptotic value due to slower diffusion rates.
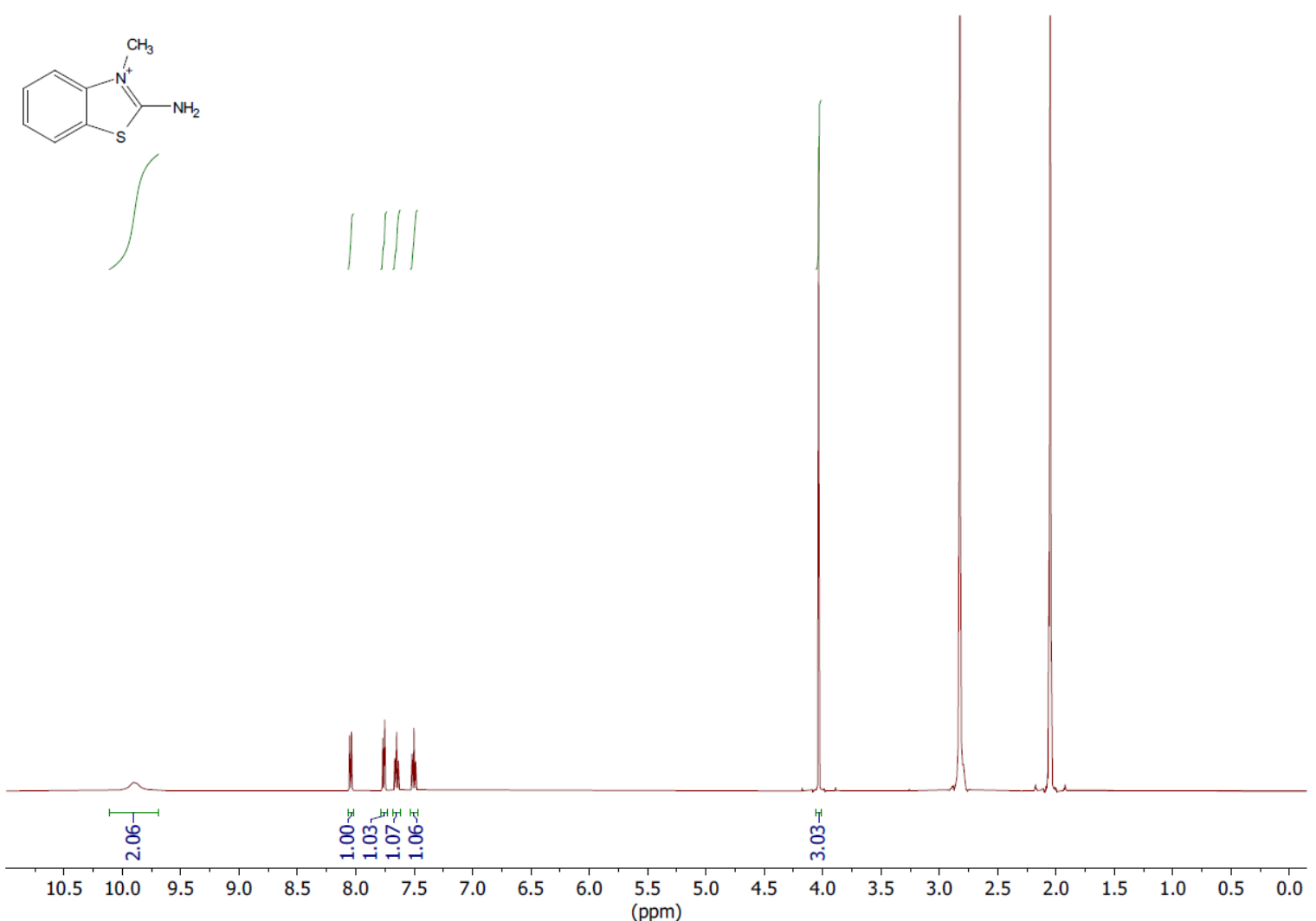

Figure S12. ${ }^{1} \mathrm{H}$ NMR spectrum of amine 1. 


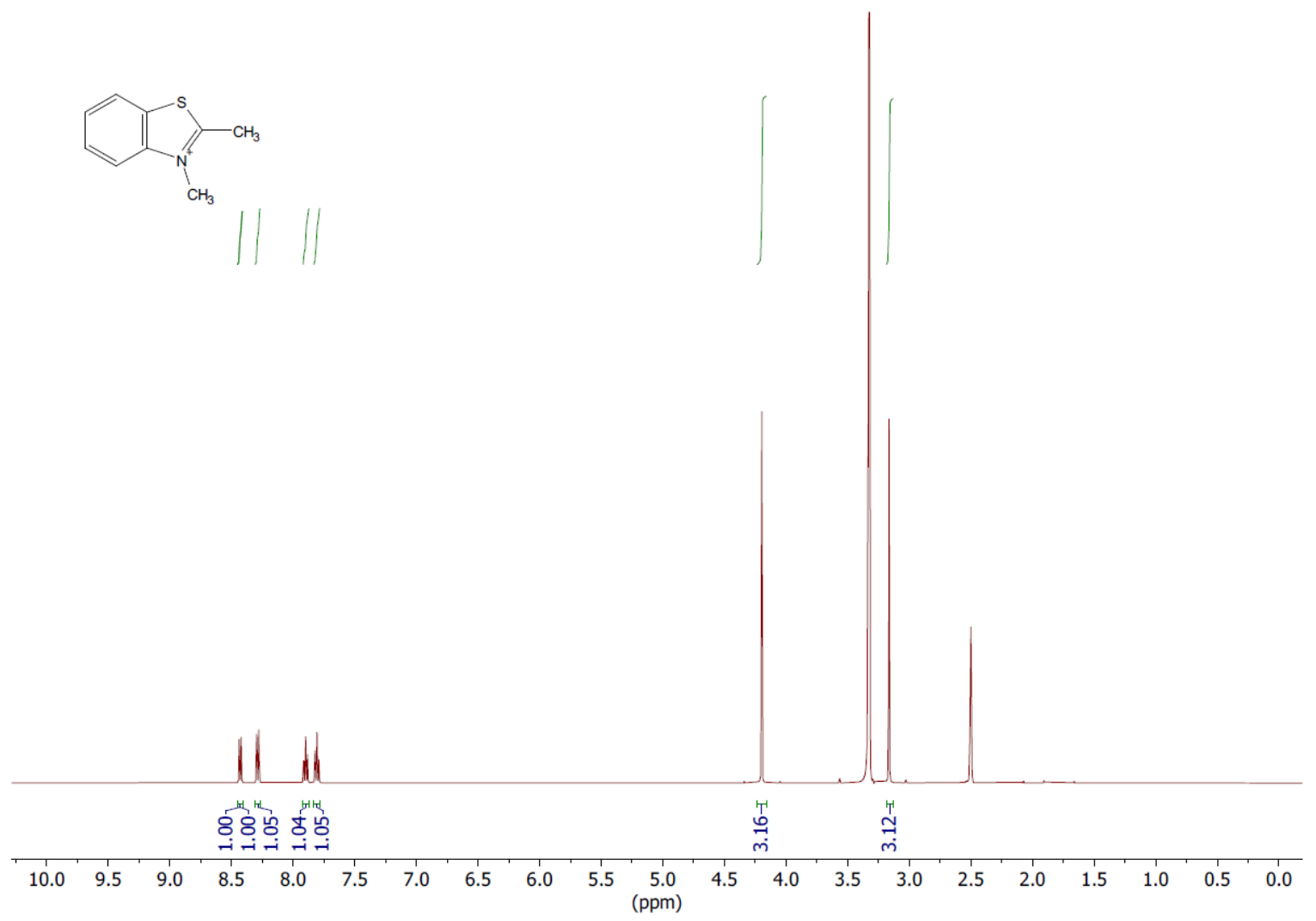

Figure S13. ${ }^{1} \mathrm{H}$ NMR spectrum of molecule $4 \mathbf{a}$.

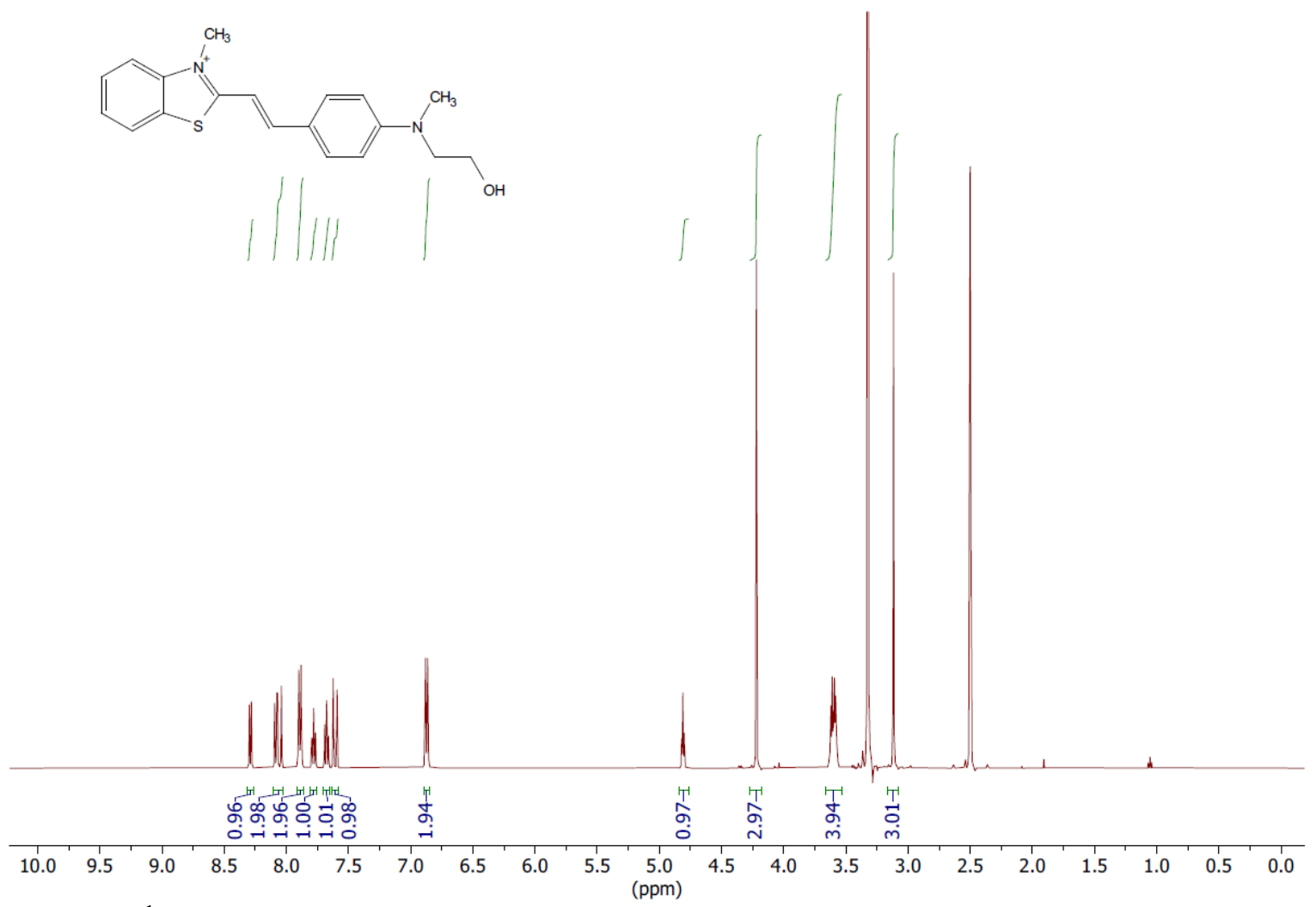

Figure S14. ${ }^{1} \mathrm{H}$ NMR spectrum of dye 4 . 
Table S1. Calculated $\log \left(\mathrm{K}_{\text {ow }}\right)$ of imine synthesis reactants and analogs. Calculated using ALGOPS 2.1.9

\begin{tabular}{c|c} 
Molecule & Estimated $\log \left(\mathbf{K}_{\mathbf{o w}}\right)$ \\
\hline Amine 1 & -1.95 \\
Aldehyde 2 & 0.97 \\
Imine 3 & -0.46 \\
Cyanine dye 4 & -0.49
\end{tabular}

Table S2. Kinetic and transport parameters used in the coacervate droplet reaction-diffusion model.

\begin{tabular}{|c|c|c|c|c|c|c|c|c|}
\hline $\begin{array}{c}{[\mathrm{NaCl}]} \\
(\mathrm{M})\end{array}$ & $\begin{array}{c}K_{\text {rxn,aq }} \\
\left(\times 10^{-3}\right. \\
\left.M^{-1}\right)\end{array}$ & $\begin{array}{c}\mathbf{k}_{\mathbf{f}, \mathbf{a q}}\left(\times \mathbf{1 0} 0^{-5}\right. \\
\left.\mathbf{M}^{-1} \mathbf{s}^{-1}\right)\end{array}$ & $\begin{array}{c}K_{\text {rxn,coac }} \\
\left(\times 10^{-3}\right. \\
\left.\mathbf{M}^{-1}\right)\end{array}$ & $\begin{array}{c}\mathbf{k f , c o a c} \\
\left(\times 10^{-5} \mathbf{M}^{-1}\right. \\
\left.\mathbf{s}^{-1}\right)\end{array}$ & $\begin{array}{c}D_{\text {im }} \\
\left(\times 10^{-12}\right. \\
\left.\mathbf{m}^{2} \mathbf{s}^{-1}\right)\end{array}$ & $\mathbf{K}_{\text {part,am }}$ & $\mathbf{K}_{\text {part,ald }}$ & $K_{\text {part,im }}$ \\
\hline$\overline{0}$ & 0.98 & 0.86 & $2.54^{\mathrm{a}}$ & $1.75^{\mathrm{a}}$ & 1.47 & 9.2 & 5.3 & 137 \\
\hline 0.2 & & & & & 1. & 4.8 & 7.5 & 86 \\
\hline 0.4 & 1.20 & 1.09 & $2.54^{\mathrm{a}}$ & $1.75^{\mathrm{a}}$ & 1.30 & 4.6 & 10.0 & 80 \\
\hline 0.6 & 1.38 & 1.34 & $2.54^{\mathrm{a}}$ & $1.75^{\mathrm{a}}$ & 1.13 & 5.1 & 12.8 & 94 \\
\hline 0.8 & 1.46 & 1.36 & $2.54^{\mathrm{a}}$ & $1.75^{\mathrm{a}}$ & 1.02 & 5.8 & 15.6 & 107 \\
\hline 1.0 & 1.48 & 1.31 & $2.54^{\mathrm{a}}$ & $1.75^{\mathrm{a}}$ & 0.95 & 5.5 & 15.8 & 104 \\
\hline
\end{tabular}

${ }^{\mathrm{a}}$ Average of $\mathrm{K}_{\text {coac }}$ and $\mathrm{k}_{\mathrm{f}, \text { coac }}$ across all $[\mathrm{NaCl}]$ shown in Figure 2 